\title{
Major Vascular Resection and Prosthetic Replacement for Retroperitoneal Tumors
}

\author{
Philipp Fueglistaler, MD, ${ }^{1}$ Lorenz Gurke, MD, ${ }^{1}$ Peter Stierli, MD, ${ }^{1}$ Tamim Obeid, MD, ${ }^{1}$ \\ Christoph Koella, MD, ${ }^{2}$ Daniel Oertli, MD, ${ }^{3}$ Christoph Kettelhack, MD ${ }^{3}$ \\ ${ }^{1}$ University Centre for Vascular Surgery, Aarau/Basel, Spitalstrasse 21, 4031 Basel, Switzerland \\ ${ }^{2}$ Department of General and Vascular Surgery, Kantonsspital Bruderholz, 4101 Bruderholz, Basel, Switzerland \\ ${ }^{3}$ Department of General Surgery, University Hospital Basel, Spitalstrasse 21, 4031 Basel, Switzerland
}

\begin{abstract}
Introduction: Involvement of major vascular structures has been considered a limiting factor for resecting advanced tumors. The objective of this study was to evaluate the outcome after concomitant retroperitoneal tumor and vascular resection with prosthetic replacement of the aorta/ vena cava.

Methods: The authors reviewed a 5-year series of eight patients with a median age of 50 years (range 11-68 years) who had undergone resection of a retroperitoneal tumor and concomitant resection and replacement of the abdominal aorta, inferior vena cava, or both. The histologic diagnoses were sarcoma (five patients), teratoma (one), transitional cell carcinoma (one), and ganglioneuroma (one). The main outcome measures were early ( $<30$ days) and late ( $\geq 30$ days) surgical morbidity and mortality. Secondary endpoints were vascular graft patency and tumor-free survival. Two patients underwent combined graft replacement of the aorta and vena cava. Single aortic and vena cava graft replacement were each done in three patients.

Results: Two patients showed early surgical morbidity necessitating reoperation for a thrombotic graft occlusion. No patient died during the early course of the follow-up. During a median follow-up of 14 months (range 1-56 months), two patients had late surgical morbidity. The median tumorfree survival for patients with malignancy was 14 months (range 1-54 months). One patient developed locoregional tumor recurrence, and two developed distant metastases. The median survival for patients with malignancy was 14 months (range 1-60 months).

Conclusions: An aggressive surgical approach for otherwise unresectable retroperitoneal tumors with vascular resection and prosthetic vascular replacement is justified in selected cases and has acceptable morbidity and mortality.
\end{abstract}

O wing to the absence of early symptoms, retroperitoneal tumors are often not diagnosed until the disease is at an advanced stage with a large tumor and involvement of surrounding structures. The involvement

This work was presented at the 122nd Congress of the German Surgical Society, Munich, Germany, March 2005 and at the International Surgical Week, Durban, South Africa, 2005.

Correspondence to: C. Kettelhack, M.D., Department of General Surgery, University Hospital Basel, Spitalstrasse 21, 4031 Basel, Switzerland, e-mail: ckettelhack@uhbs.ch of major vascular structures has traditionally been considered a limiting factor or even a relative contraindication to resection of advanced tumors because of the poor long-term prognosis and high surgical risk. Nevertheless, during recent decades several studies have shown promising results after concomitant tumor and vascular resection at various localizations. ${ }^{1-7}$ The aorta and the vena cava have rarely been replaced in patients with malignancy because of the magnitude and high risk of the operation. ${ }^{1}$ The operative treatment of tumors involving 
the aorta or vena cava (or both) has been improved by the introduction of polytetrafluoroethylene (PTFE) grafts for vascular reconstruction. ${ }^{8}$ Even so, the surgery needed to achieve complete tumor resection is challenging.

Determining the utility of retroperitoneal tumor resection including resection and prosthetic replacement of the aorta/vena cava is difficult because few studies have dealt with the vascular complications associated with major arterial or venous procedures and simultaneous tumor resection. . $^{1,2,9,10}$

The aim of this study was to determine whether this extended surgical procedure is justified. We reviewed the outcomes of patients who underwent concomitant retroperitoneal tumor resection and vascular resection combined with prosthetic replacement of the abdominal aorta or inferior vena cava (or both).

\section{MATERIALS AND METHODS}

We reviewed the records of eight patients (four women) with a median age of 50 years (range 11-68 years) treated from September 1999 to September 2004 by an oncologic procedure for vascular involvement of a retroperitoneal neoplasm requiring concomitant resection of the infrarenal aorta or inferior vena cava (or both) with a vascular replacement prosthesis (Fig. 1). Exclusion criteria were vascular resection without prosthetic reconstruction, reconstruction of the superior vena cava and thoracic aorta, and renal cell carcinoma requiring only removal of a tumor thrombus in the vena cava.

Long-term follow-up was obtained in seven of eight patients. One patient (Table 1) was lost to follow-up 1 month after the operation. The main outcome measures were early ( $<30$ days) and late ( $\geq 30$ days) surgical morbidity and mortality. Secondary endpoints were vascular graft patency and tumor-free survival. Perioperative and postoperative morbidity/mortality, surgical procedure, and histopathologic diagnosis of the tumor were identified by a review of medical records and pathology reports. The patency of the vascular replacement was evaluated by a review of follow-up flow duplex imaging, computed tomographic (CT) scans, or clinical examination. Survival and tumor recurrence were determined by a review of medical reports.

Single aortic or vena cava graft replacement was each done in three patients (Table 1). Two patients underwent combined graft replacement of the aorta and vena cava (Fig. 2). For vascular prosthetic replacement a PTFE graft was used in all patients. Aortic prosthetic replacement (diameters 8-16 mm) included only the infrarenal aorta.

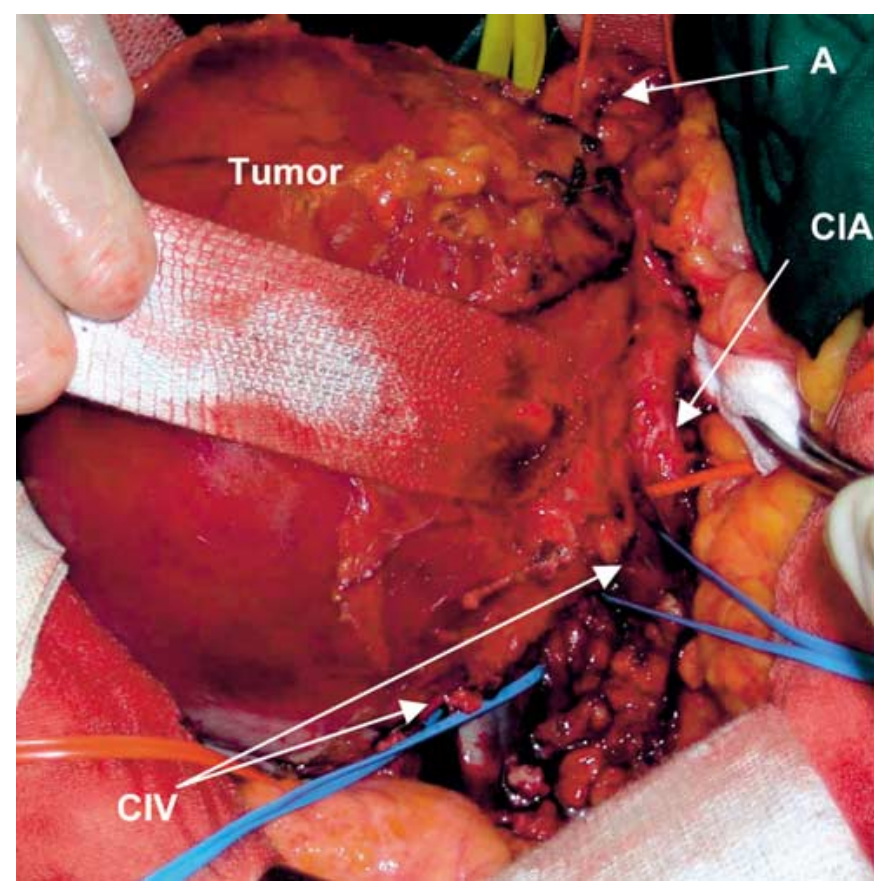

Figure 1. Intraoperative view of the liposarcoma with infiltration of aorta and vena cava. CIA: common iliac arteries; CIV: common iliac veins; $A$ : aorta.

Vena cava prosthetic replacement (diameters 16-20 mm) was performed below the renal veins in all but two patients. One patient underwent reimplantation of the left renal vein.

Vascular resection and replacement were done without extracorporeal circulation in all patients. Intraoperative heparin sodium was routinely administered before vascular clamping. Postoperatively, partial thromboplastin time-controlled heparin sodium was administered to five patients and low-molecular-weight heparin to three patients for 5 days. Subsequently, Coumarin anticoagulant was given to four patients and chronic antiplatelet therapy to the other four. The vascular replacement procedures were performed electively in conjunction with tumor resection in six of eight patients. Vascular replacement was required as an emergency procedure in two patients because of a vascular injury that occurred during resective oncologic surgery.

The tumor was malignant in seven of the eight patients. The pathologic diagnosis was sarcoma in five patients, teratoma in one, transitional cell carcinoma in one, and benign ganglioneuroma in one (Table 1). In seven of the eight patients, histopathologic examination revealed direct vascular infiltration by the tumor (Fig. 3). Negative margin resection $(\mathrm{R} 0)$ was achieved in four patients and positive microscopic/negative gross margin resection (R1) in three. Only one of the patients (patient 6) showed a positive microscopic margin (R1) in the resected vascular structure; in two other patients microscopic tumor 

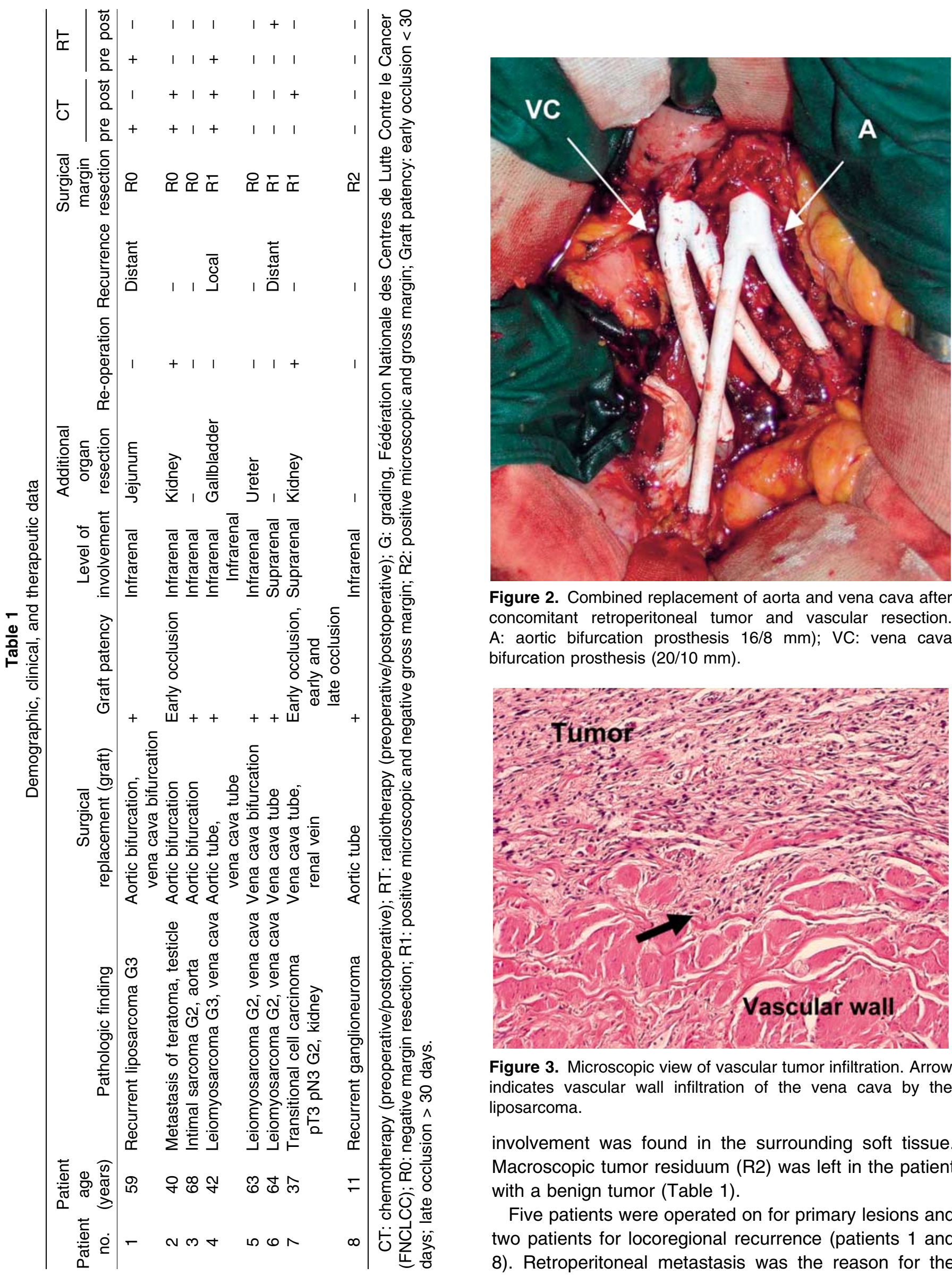

Figure 2. Combined replacement of aorta and vena cava after concomitant retroperitoneal tumor and vascular resection. A: aortic bifurcation prosthesis $16 / 8 \mathrm{~mm}$ ); VC: vena cava bifurcation prosthesis $(20 / 10 \mathrm{~mm})$.

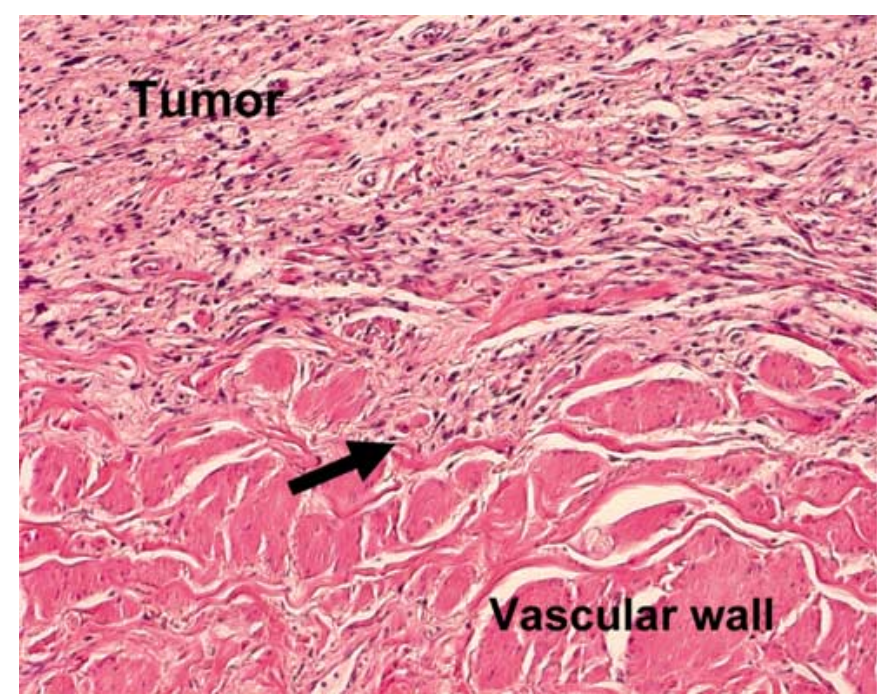

Figure 3. Microscopic view of vascular tumor infiltration. Arrow indicates vascular wall infiltration of the vena cava by the liposarcoma.

involvement was found in the surrounding soft tissue. Macroscopic tumor residuum (R2) was left in the patient with a benign tumor (Table 1).

Five patients were operated on for primary lesions and two patients for locoregional recurrence (patients 1 and 8). Retroperitoneal metastasis was the reason for the 


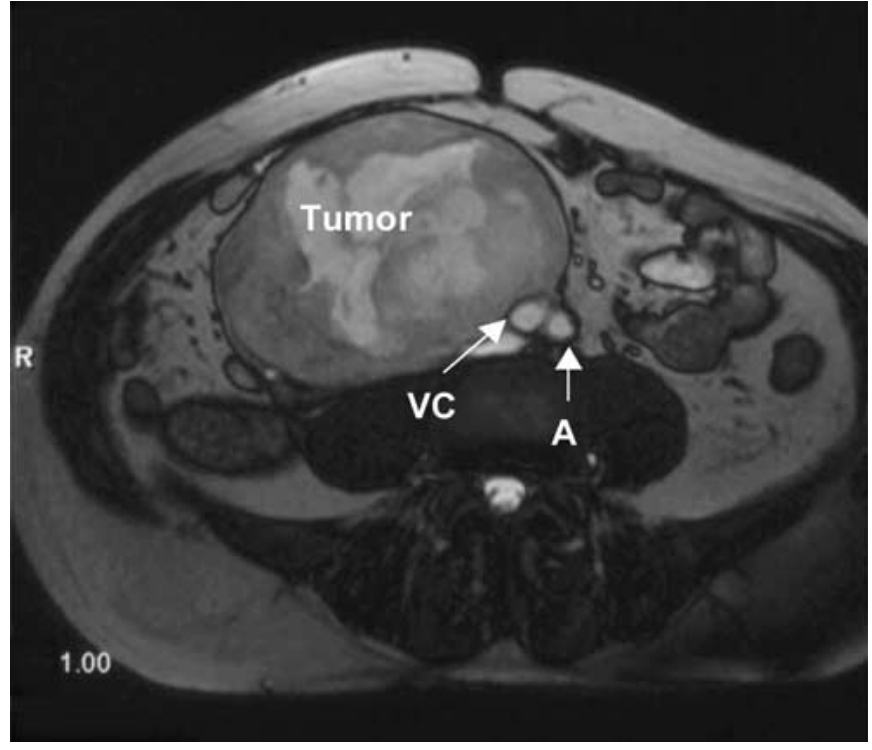

Figure 4. Axial magnetic resonance image demonstrates vascular infiltration by the retroperitoneal liposarcoma. A: aorta; VC: vena cava.

operation in one patient (patient 2). One patient (patient 3) was operated on 2 months after prosthetic replacement of an aortic aneurysm because the histopathologic examination revealed an intimal sarcoma of the aorta. Resection of contiguous organs was performed in five patients (Table 1).

The preoperative clinical performance status was defined according to the American Society of Anesthesiologists (ASA) classification: Three patients were classified as ASA 2 and five as ASA 3. The mean operating time was 340 minutes. Seven patients required blood transfusion perioperatively (median requirement 4 units; range 0-15 units). Preoperatively, two patients underwent combined chemotherapy/radiotherapy, and one had chemotherapy only (Table 1). Postoperatively, chemotherapy was administered to three patients and radiotherapy to one. Preoperative diagnostic evaluation included chest radiography, CT scan of the abdomen, or magnetic resonance imaging (or a combination of these tests) in all patients and CT- or ultrasound-guided biopsy in five patients (Fig. 4). Perioperatively, all patients were given antibiotic prophylaxis with a second-generation cephalosporin for 24 hours.

\section{RESULTS}

Two of eight patients showed early surgical morbidity after being reoperated for a thrombotic graft occlusion. The patient (patient 7) operated on for vena cava replacement and reimplantation of the left renal vein had to be reoperated 24 hours later for a graft thrombectomy and prosthetic reconstruction of the left renal vein. This patient also developed esophagitis and kidney failure requiring hemodialysis. Another patient (patient 2) was reoperated for a graft bifurcation thrombectomy 6 hours after aortic replacement. The latter patient had a pulmonary infection and Clostridium difficile colitis. Two patients (patients 5 and 8 ) developed urinary infections, causing pyelonephritis in one patient (patient 5). None of the patients died as a result of the surgical procedure.

During a median follow-up of 14 months (range 1-56 months; mean 18 months), two of seven patients had late surgical morbidity. One patient (patient 5) was reoperated 4 months after replacement of the vena cava and resection of the right tumor-involved ureter; nephrectomy was done for ureteral leakage that caused a urinoma.

One patient (patient 7) underwent CT-guided drainage of a retroperitoneal hematoma 1 month postoperatively. This patient also had a thrombotic occlusion of the left renal vein graft 2 months after prosthetic replacement of the vena cava and left renal vein. Because kidney function was normal, reoperation was not indicated. There were no reported graft infections.

The median tumor-free survival for patients with malignancy was 14 months (range 1-54 months). One patient developed locoregional tumor recurrence (patient 4) and died of it 11 months after operation. Two patients developed distant metastases (patients 1 and 6). One of these patients (patient 6) had an abdominal wall (incisional) metastasis necessitating abdominal wall resection after 22 months. The other patient (patient 1) developed several metastases in the lung. The median survival for patients with malignancy was 14 months (range 1-60 months).

Graft patency failure occurred in two patients (patients 2 and 7) who were reoperated during the early follow-up. Patient 7 also developed a thrombotic occlusion of the left renal vein graft 2 months postoperatively, as described above.

\section{DISCUSSION}

Patients who undergo concomitant resection of a retroperitoneal tumor and major vascular structures usually exhibit considerable perioperative morbidity because of the extent of the surgical procedure. In addition, these patients may be in poor general condition as a result of advanced disease and sometimes preoperative chemotherapy. In this study only two patients showed vascular 
problems that required reoperation, and no sequelae resulted. Nonvascular complications during the early course of follow-up were minor. No perioperative deaths occurred, underlining the feasibility of this technically demanding surgical procedure in carefully selected patients. ${ }^{1,10-13}$ In contrast, one study showed an early morbidity incidence of $44 \%$ after vena cava replacement, including major complications such as intraoperative coagulopathy with graft occlusion and graft infection leading to death. ${ }^{1}$ In another series similar to the present one but using different vascular procedures, the early morbidity was $19 \%$ in patients with aortic and vena cava replacement. ${ }^{10}$

During the median follow-up of 14 months, both median tumor-free survival and median survival for the seven patients with malignancies were 14 months; only one patient died after 11 months. The small number of patients, the different types of tumor, and the rather short follow-up period precluded definitive conclusions about the impact of these operations on survival. Moreover, the present study was primarily concerned with the rationale for, and the feasibility of, a combined major surgical approach as part of extended therapeutic management.

Vascular intervention may be necessary to cure or palliate symptoms by concomitant resection and replacement of major vessels infiltrated by a tumor. Alternatively, a rescue procedure may be necessary because of an intraoperative vascular injury, as was the case in two of our patients. Precise preoperative planning of the surgical approach is important for complete tumor resection combined with low perioperative morbidity. In the case of sarcomas (the main tumor type in this study), complete tumor resection is the main predictor of a low rate of tumor recurrence and increased survival. ${ }^{11,14-16}$ In a series of 500 patients with retroperitoneal sarcoma, the survival rate was significantly reduced in patients whose resection was incomplete, ${ }^{17}$ but other series have found no long-term survivors even after complete resection of retroperitoneal sarcoma with major vascular resection. ${ }^{9,18}$ For retroperitoneal sarcoma, complete tumor resection is reported in $59 \%$ to $90 \%$ of patients, with a higher rate for primary tumors compared with recurrent tumors. ${ }^{11,15,17,19,20}$ In the present series, complete resection (R0) was achieved in three of the five patients with sarcoma, one of whom was operated on for a recurrent tumor.

Three of seven patients with malignancy developed recurrence, giving a median tumor-free survival of 14 months, which is consistent with earlier findings of tumor recurrence in more than $50 \%$ of patients after prosthetic replacement of the vena cava for malignancy during a median follow-up of 16 months. ${ }^{21}$
A contiguous organ was resected in more than half of our patients, consistent with other series of retroperitoneal sarcoma, who have reported additional organ resection in $44 \%$ to $93 \% .^{15-17,21,22}$ For retroperitoneal tumors, multivisceral resection is done not only to achieve negative surgical margins but also to facilitate the surgical procedure technically. ${ }^{21}$ One study found histopathologic confirmation of tumor involvement in only $4 \%$ of resected organs. ${ }^{16}$

Nearly all of our patients received multimodal treatment, depending on tumor biology and tumor involvement of surgical margins (Table 1). Although some studies have shown reduced disease recurrence or even improved patient survival after radiotherapy, ${ }^{11,14,15,17}$ multimodal treatment for the management of retroperitoneal sarcoma remains controversial. This is because the radiation dose is limited by the tolerance of the surrounding structures, the volume of the retroperitoneum to be irradiated is high. ${ }^{23-25}$ Uncertain effects have been demonstrated for chemotherapy alone. ${ }^{26-28}$

After vascular replacement, 3 of 11 grafts developed thrombotic occlusion and required reoperation. The patency of the prosthetic replacement was evaluated by imaging in five patients. We interpret the lack of clinical symptoms of graft occlusion in the others as evidence of a patent replacement because occlusion of a major retroperitoneal vascular replacement usually becomes symptomatic.

Our general approach includes intravenous administration of heparin sodium immediately after aortic and vena cava graft replacement. This is followed by postoperative lifelong chronic antiplatelet therapy in patients with an aortic graft. Oral anticoagulation of undetermined duration is given after vena cava replacement. However, in the present series with concomitant resection of tumors, individual tailoring of treatment according to an interdisciplinary discussion resulted in postoperative administration of low-molecular-weight heparin alone in three patients. In this series there were no other graftrelated complications, nor were there any later deaths or complications as a result of vascular patency failure.

\section{CONCLUSIONS}

We believe that these results justify an extended surgical approach to retroperitoneal neoplasms, including prosthetic vascular replacement, because early morbidity and mortality were low and graft patency did not limit the late course of the disease. We also believe that prosthetic replacement of the infrarenal aorta or inferior vena cava 
(or both) is a safe option with few graft-related complications. Because it provides the only chance of controlling advanced retroperitoneal tumors, we favor this aggressive surgical management.

\section{REFERENCES}

1. Bower TC, Nagorney DM, Cherry KJ, et al. Replacement of the inferior vena cava for malignancy: an update. J Vasc Surg 2000;31:270-281.

2. Caldarelli G, Minervini A, Guerra M, et al. Prosthetic replacement of the inferior vena cava and the iliofemoral vein for urologically related malignancies. BJU Int 2002;90: 368-374.

3. Harrison LE, Klimstra DS, Brennan MF. Isolated portal vein involvement in pancreatic adenocarcinoma: a contraindication for resection? Ann Surg 1996;224:342-347.

4. Hemming AW, Reed Al, Langham MR, et al. Combined resection of the liver and inferior vena cava for hepatic malignancy. Ann Surg 2004;239:712-721.

5. Hohenberger P, Allenberg JR, Schlag PM, et al. Results of surgery and multimodal therapy for patients with soft tissue sarcoma invading to vascular structures. Cancer 1999;85: 396-408.

6. Koperna T, Teleky B, Vogl S, et al. Vascular reconstruction for limb salvage in sarcoma of the lower extremity. Arch Surg 1996;131:1103-1107.

7. Wright JG, Nicholson R, Schuller DE, et al. Resection of the internal carotid artery and replacement with greater saphenous vein: a safe procedure for en bloc cancer resections with carotid involvement. J Vasc Surg 1996;23: 775-780.

8. Gloviczki P, Pairolero PC, Toomey BJ, et al. Reconstruction of large veins for nonmalignant venous occlusive disease. J Vasc Surg 1992;16:750-761.

9. Bianchi C, Ballard JL, Bergan JH, et al. Vascular reconstruction and major resection for malignancy. Arch Surg 1999;134:851-855.

10. Di Perna CA, Bowdish ME, Weaver FA, et al. Concomitant vascular procedures for malignancies with vascular invasion. Arch Surg 2002;137:901-907.

11. Ferrario T, Karakousis CP. Retroperitoneal sarcomas. Arch Surg 2003;138:248-251.

12. Huguet C, Ferri M, Gavelli A. Resection of the suprarenal inferior vena cava: the role of prosthetic replacement. Arch Surg 1995;130:793-797.
13. Sarkar R, Eilber FR, Gelabert HA, et al. Prosthetic replacement of the inferior vena cava for malignancy. J Vasc Surg 1998;28:75-83.

14. Karakousis CP, Gerstenbluth R, Kontzoglou K, et al. Retroperitoneal sarcomas and their management. Arch Surg 1995;130:1104-1109.

15. Kilkenny JW, Bland KI, Copeland EM. Retroperitoneal sarcoma: the University of Florida experience. J Am Coll Surg 1996;182:329-339.

16. Neuhaus SJ, Barry P, Clark MA, et al. Surgical management of primary and recurrent retroperitoneal liposarcoma. Br J Surg 2005;92:246-252.

17. Lewis JJ, Leung D, Woodruff JM, et al. Retroperitoneal softtissue sarcoma: analysis of 500 patients treated and followed at a single institution. Ann Surg 1998;228:355-365.

18. Bevilacqua RG, Rogatko A, Hajdu SI, et al. Prognostic factors in primary retroperitoneal soft-tissue sarcomas. Arch Surg 1991;126:328-334.

19. Mäkelä J, Kiviniemi H, Laitinen S. Prognostic factors predicting survival in the treatment of retroperitoneal sarcoma. Eur J Surg Oncol 2000;26:552-555.

20. Zornig C, Weh HJ, Krull A, et al. Retroperitoneal sarcoma in a series of 51 adults. Eur J Surg Oncol 1992;18:475-480.

21. Hardwigsen J, Baqué $P$, Crespy $B$, et al. Resection of the inferior vena cava for neoplasms with or without prosthetic replacement: a 14-patient series. Ann Surg 2001;2:242249.

22. Nagel M, Ockert D, Stoelben E, et al. Results of surgical therapy of soft tissue sarcoma of the retroperitoneum. Zentralbl Chir 1994;119:488-494.

23. Pisters PW, Ballo MT, Shreyaskumar RP. Preoperative chemoradiation treatment strategies for localized sarcoma. Ann Surg Oncol 2002;9:535-542.

24. Hassan I, Park SZ, Donohue JH, et al. Operative management of primary retroperitoneal sarcomas: a reappraisal of an institutional experience. Ann Surg 2004;239:244-250.

25. Pirayesh A, Chee $Y$, Helliwell TR, et al. The management of retroperitoneal soft tissue sarcoma: a single institution experience with a review of the literature. Eur J Surg Oncol 2001;27:491-497.

26. Adjuvant chemotherapy for localised resectable soft tissue sarcoma in adults: Sarcoma Meta-analysis Collaboration (SMAC). Cochrane Database Syst Rev 2000;2:CD001419.

27. Lewis JJ, Benedetti F. Adjuvant therapy for soft tissue sarcomas. Surg Oncol Clin North Am 1997;6:847-862.

28. Singer S, Corson JM, Demetri GD, et al. Prognostic factors predictive of survival for truncal and retroperitoneal softtissue sarcoma. Ann Surg 1995;221:185-195. 